\title{
Justify your alpha
}

In response to recommendations to redefine statistical significance to $P \leq 0.005$, we propose that researchers should transparently report and justify all choices they make when designing a study, including the alpha level.

Daniel Lakens, Federico G. Adolfi, Casper J. Albers, Farid Anvari, Matthew A. J. Apps, Shlomo E. Argamon, Thom Baguley, Raymond B. Becker, Stephen D. Benning, Daniel E. Bradford, Erin M. Buchanan, Aaron R. Caldwell, Ben Van Calster, Rickard Carlsson, Sau-Chin Chen, Bryan Chung, Lincoln J. Colling, Gary S. Collins, Zander Crook, Emily S. Cross, Sameera Daniels, Henrik Danielsson, Lisa DeBruine, Daniel J. Dunleavy, Brian D. Earp, Michele I. Feist, Jason D. Ferrell, James G. Field, Nicholas W. Fox, Amanda Friesen, Caio Gomes, Monica Gonzalez-Marquez, James A. Grange, Andrew P. Grieve, Robert Guggenberger, James Grist, Anne-Laura van Harmelen, Fred Hasselman, Kevin D. Hochard, Mark R. Hoffarth, Nicholas P. Holmes, Michael Ingre, Peder M. Isager, Hanna K. Isotalus, Christer Johansson, Konrad Juszczyk, David A. Kenny, Ahmed A. Khalil, Barbara Konat, Junpeng Lao, Erik Gahner Larsen, Gerine M. A. Lodder, Jiří Lukavský, Christopher R. Madan, David Manheim, Stephen R. Martin, Andrea E. Martin, Deborah G. Mayo, Randy J. McCarthy, Kevin McConway, Colin McFarland, Amanda Q. X. Nio, Gustav Nilsonne, Cilene Lino de Oliveira, Jean-Jacques Orban de Xivry, Sam Parsons, Gerit Pfuhl, Kimberly A. Quinn, John J. Sakon, S. Adil Saribay, Iris K. Schneider, Manojkumar Selvaraju, Zsuzsika Sjoerds, Samuel G. Smith, Tim Smits, Jeffrey R. Spies, Vishnu Sreekumar, Crystal N. Steltenpohl, Neil Stenhouse, Wojciech Świątkowski, Miguel A. Vadillo, Marcel A. L. M. Van Assen, Matt N. Williams, Samantha E. Williams, Donald R. Williams, Tal Yarkoni, Ignazio Ziano and Rolf A. Zwaan

$\mathrm{B}$ enjamin et al. ${ }^{1}$ proposed changing the conventional "statistical significance" threshold (that is, the alpha level) from $P \leq 0.05$ to $P \leq 0.005$ for all novel claims with relatively low prior odds. They provided two arguments for why lowering the significance threshold would "immediately improve the reproducibility of scientific research". First, a $P$ value near 0.05 provides weak evidence for the alternative hypothesis. Second, under certain assumptions, an alpha level of 0.05 leads to high false positive report probabilities (FPRPs ${ }^{2}$; the probability that a significant finding is a false positive).

We share their concerns regarding the apparent non-replicability of many scientific studies, and agree that a universal alpha of 0.05 is undesirable. However, redefining "statistical significance" to a lower, but equally arbitrary threshold, is inadvisable for three reasons: (1) there is insufficient evidence that the current standard is a "leading cause of non-reproducibility"; (2) the arguments in favour of a blanket default of $P \leq 0.005$ do not warrant the immediate and widespread implementation of such a policy; and (3) a lower significance threshold will likely have negative consequences not discussed by Benjamin and colleagues. We conclude that the term "statistically significant" should no longer be used and suggest that researchers employing null hypothesis significance testing justify their choice for an alpha level before collecting the data, instead of adopting a new uniform standard.

$P \leq 0.005$ does not improve replicability Benjamin et al. ${ }^{1}$ claimed that the expected proportion of replicable studies should be considerably higher for studies observing $P \leq 0.005$ than for studies observing $0.005<$ $P \leq 0.05$, due to a lower FPRP. Theoretically, replicability is related to the FPRP, and lower alpha levels will reduce false positive results in the literature. However, in practice, the impact of lowering alpha levels depends on several unknowns, such as the prior odds that the examined hypotheses are true, the statistical power of studies and the (change in) behaviour of researchers in response to any modified standards.

An analysis of the results of the Reproducibility Project: Psychology ${ }^{3}$ showed that $49 \%(23 / 47)$ of the original findings with $P$ values below 0.005 yielded $P \leq 0.05$ in the replication study, whereas only $24 \%(11 / 45)$ of the original studies with $0.005<P \leq 0.05$ yielded $P \leq 0.05$ $\left(\chi^{2}(1)=5.92, P=0.015\right.$, Bayes factor $\left.\mathrm{BF}_{10}=6.84\right)$. Benjamin and colleagues presented this as evidence of "potential gains in reproducibility that would accrue from the new threshold". According to their own proposal, however, this evidence is only "suggestive" of such a conclusion, and there is considerable variation in replication rates across $P$ values (see Fig. 1). Importantly, lower replication rates for $P$ values just below 0.05 are likely confounded by $P$-hacking (the practice of flexibly analysing data until the $P$ value passes the "significance" threshold). Thus, the differences in replication rates between studies with $0.005<P \leq 0.05$ compared with those with $P \leq 0.005$ may not be entirely due to the level of evidence. Further analyses are needed to explain the low (49\%) replication rate of studies with $P \leq 0.005$, before this alpha level is recommended as a new significance threshold for novel discoveries across scientific disciplines.

\section{Weak justifications for $\alpha=0.005$} We agree with Benjamin et al. that single $P$ values close to 0.05 never provide strong "evidence" against the null hypothesis. Nonetheless, the argument that $P$ values provide weak evidence based on Bayes factors has been questioned ${ }^{4}$. Given that the marginal likelihood is sensitive to different choices for the models being compared, redefining alpha levels as a function of the Bayes factor is undesirable. For instance, Benjamin and colleagues stated that $P$ values of 0.005 imply Bayes factors between 14 and 26. However, these upper bounds only hold for a Bayes factor based on a point null 


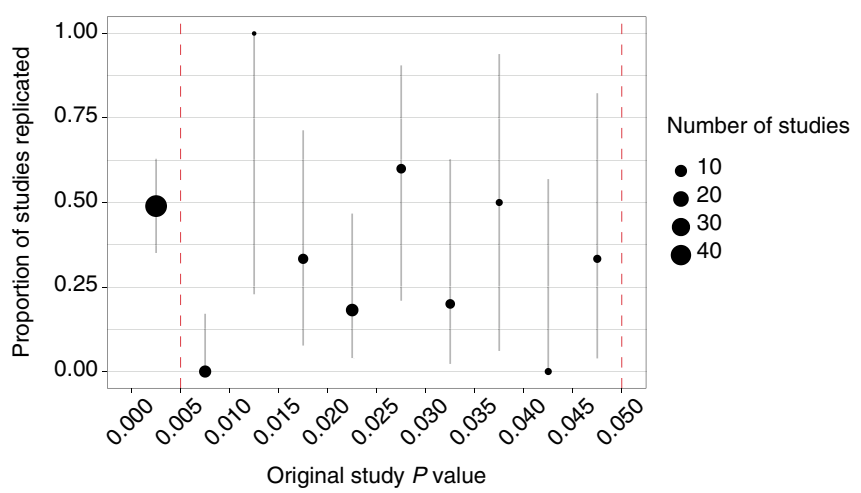

Fig. 1 | The proportion of studies ${ }^{3}$ replicated at $\alpha=0.05$ (with a bin width of 0.005 ). Window start and end positions are plotted on the horizontal axis. Red dashed lines indicate 0.005 and 0.05 thresholds. The error bars denote $95 \%$ Jeffreys confidence intervals. R code to reproduce Fig. 1 is available from https://osf.io/by2kc/.

model and when the $P$ value is calculated for a two-sided test, whereas one-sided tests or Bayes factors for non-point null models would imply different alpha thresholds. When a test yields $\mathrm{BF}=25$, the data are interpreted as strong relative evidence for a specific alternative (for example, mean $=2.81$, while a $P \leq 0.005$ only warrants the more modest rejection of a null effect without allowing one to reject even small positive effects with a reasonable error rate ${ }^{5}$. Benjamin et al. provided no rationale for why the new $P$ value threshold should align with equally arbitrary Bayes factor thresholds. We question the idea that the alpha level at which an error rate is controlled should be based on the amount of relative evidence indicated by Bayes factors.

The second argument for $\alpha=0.005$ is that the FPRP can be high with $\alpha=0.05$. Calculating the FPRP requires a definition of the alpha level, the power of the tests examining true effects and the ratio of true to false hypotheses tested (the prior odds). Figure 2 in Benjamin et al. shows FPRPs for scenarios where most hypotheses are false, with prior odds of 1:5, 1:10 and 1:40. The recommended $P \leq 0.005$ threshold reduces the minimum FPRP to less than $5 \%$, assuming 1:10 prior odds (the true FPRP might still be substantially higher in studies with very low power). This prior odds estimate is based on data from the Reproducibility Project: Psychology ${ }^{3}$ using an analysis modelling publication bias for 73 studies $^{6}$. Without stating the reference class for the "base rate of true nulls"

(for example, does this refer to all hypotheses in science, in a discipline or by a single researcher?), the concept of "prior odds that the alternative hypothesis is true" has little meaning. Furthermore, there is insufficient representative data to accurately estimate the prior odds that researchers examine a true hypothesis, and thus, there is currently no strong argument based on FPRP to redefine statistical significance.

\section{$P \leq 0.005$ might harm scientific practice}

Benjamin et al. acknowledged that their proposal has strengths as well as weaknesses, but believe that its "efficiency gains would far outweigh losses." We are not convinced and see at least three likely negative consequences of adopting a lowered threshold.

Risk of fewer replication studies. All else being equal, lowering the alpha level requires larger sample sizes and creates an even greater strain on already limited resources. Achieving $80 \%$ power with $\alpha=0.005$, compared with $\alpha=0.05$, requires a 70\% larger sample size for between-subjects designs with two-sided tests ( $88 \%$ for onesided tests). While Benjamin et al. propose $\alpha=0.005$ exclusively for "new effects" (and not replications), designing larger original studies would leave fewer resources (that is, time, money, participants) for replication studies, assuming fixed resources overall. At a time when replications are already relatively rare and unrewarded, lowering alpha to 0.005 might therefore reduce resources spent on replicating the work of others. More generally, recommendations for evidence thresholds need to carefully balance statistical and non-statistical considerations (for example, the value of evidence for a novel claim versus the value of independent replications).

Risk of reduced generalizability and breadth. Requiring larger sample sizes across scientific disciplines may exacerbate over-reliance on convenience samples (for example, undergraduate students, online samples). Specifically, without (1) increased funding, (2) a reward system that values large-scale collaboration and (3) clear recommendations for how to evaluate research with sample size constraints, lowering the significance threshold could adversely affect the breadth of research questions examined. Compared with studies that use convenience samples, studies with unique populations (for example, people with rare genetic variants, patients with post-traumatic stress disorder) or with time- or resource-intensive data collection (for example, longitudinal studies) require considerably more research funds and effort to increase the sample size. Thus, researchers may become less motivated to study unique populations or collect difficultto-obtain data, reducing the generalizability and breadth of findings.

Risk of exaggerating the focus on single $\boldsymbol{P}$ values. The proposal of Benjamin et al. risks (1) reinforcing the idea that relying on $P$ values is a sufficient, if imperfect, way to evaluate findings and (2) discouraging opportunities for more fruitful changes in scientific practice and education. Even though Benjamin et al. do not propose $P \leq 0.005$ as a publication threshold, some bias in favour of significant results will remain, in which case redefining $P \leq 0.005$ as "statistically significant" would result in greater upward bias in effect size estimates. Furthermore, it diverts attention from the cumulative evaluation of findings, such as converging results of multiple (replication) studies.

\section{No one alpha to rule them all}

We have two key recommendations. First, we recommend that the label "statistically significant" should no longer be used. Instead, researchers should provide more meaningful interpretations of the theoretical or practical relevance of their results. Second, authors should transparently specify - and justify - their design choices. Depending on their choice of statistical approach, these may include the alpha level, the null and alternative models, assumed prior odds, statistical power for a specified effect size of interest, the sample size, and/ or the desired accuracy of estimation. We do not endorse a single value for any design parameter, but instead propose that authors justify their choices before data are collected. Fellow researchers can then evaluate these decisions, ideally also before data collection, for example, by reviewing a registered report submission ${ }^{7}$. Providing researchers (and reviewers) with accessible information about ways to justify (and evaluate) design 
choices, tailored to specific research areas, will improve current research practices.

Benjamin et al. noted that some fields, such as genomics and physics, have lowered the "default" alpha level. However, in genomics the overall false positive rate is still controlled at 5\%; the lower alpha level is only used to correct for multiple comparisons. In physics, researchers have argued against a blanket rule, and for an alpha level based on factors such as the surprisingness of the predicted result and its practical or theoretical impact ${ }^{8}$. In nonhuman animal research, minimizing the number of animals used needs to be directly balanced against the probability and cost of false positives. Depending on these and other considerations, the optimal alpha level for a given research question could be higher or lower than the current convention of 0.05 (refs $^{9-11}$ ).

Benjamin et al. stated that a "critical mass of researchers" endorse the standard of a $P \leq 0.005$ threshold for "statistical significance". However, the presence of a critical mass can only be identified after a norm has been widely adopted, not before. Even if a $P \leq 0.005$ threshold were widely accepted, this would only reinforce the misconception that a single alpha level is universally applicable. Ideally, the alpha level is determined by comparing costs and benefits against a utility function using decision theory ${ }^{12}$. This cost-benefit analysis (and thus the alpha level) ${ }^{13}$ differs when analysing large existing datasets compared with collecting data from hardto-obtain samples.

\section{Conclusion}

Science is diverse, and it is up to scientists to justify the alpha level they decide to use. As Fisher noted"14: "No scientific worker has a fixed level of significance at which, from year to year, and in all circumstances, he rejects hypotheses; he rather gives his mind to each particular case in the light of his evidence and his ideas." Research should be guided by principles of rigorous science $^{15}$, not by heuristics and arbitrary blanket thresholds. These principles include not only sound statistical analyses, but also experimental redundancy (for example, replication, validation and generalization), avoidance of logical traps, intellectual honesty, research workflow transparency and accounting for potential sources of error. Single studies, regardless of their $P$ value, are never enough to conclude that there is strong evidence for a substantive claim. We need to train researchers to assess cumulative evidence and work towards an unbiased scientific literature. We call for a broader mandate beyond $P$-value thresholds whereby all justifications of key choices in research design and statistical practice are transparently evaluated, fully accessible and pre-registered whenever feasible.

\section{Daniel Lakens ${ }^{1 \star}$, Federico G. Adolfi' ${ }^{2,3}$, \\ Casper J. Albers ${ }^{4}$, Farid Anvari ${ }^{5}$,}

Matthew A. J. Apps ${ }^{6}$, Shlomo E. Argamon',

Thom Baguley ${ }^{8}$, Raymond B. Becker ${ }^{9}$,

Stephen D. Benning ${ }^{10}$, Daniel E. Bradford"1,

Erin M. Buchanan ${ }^{12}$, Aaron R. Caldwell'13,

Ben Van Calster ${ }^{14,15}$, Rickard Carlsson ${ }^{16}$,

Sau-Chin Chen ${ }^{17}$, Bryan Chung ${ }^{18}$,

Lincoln J. Colling ${ }^{19}$, Gary S. Collins ${ }^{20}$,

Zander Crook ${ }^{21}$, Emily S. Cross ${ }^{22,23}$,

Sameera Daniels ${ }^{24}$, Henrik Danielsson ${ }^{25}$,

Lisa DeBruine ${ }^{23}$, Daniel J. Dunleavy ${ }^{26}$,

Brian D. Earp ${ }^{27}$, Michele I. Feist ${ }^{28}$,

Jason D. Ferrell29,30, James G. Field ${ }^{31}$,

Nicholas W. Fox ${ }^{32}$, Amanda Friesen ${ }^{33}$,

Caio Gomes ${ }^{34}$, Monica Gonzalez-Marquez ${ }^{35}$,

James A. Grange ${ }^{36}$, Andrew P. Grieve ${ }^{37}$,

Robert Guggenberger ${ }^{38,39}$, James Grist ${ }^{40}$,

Anne-Laura van Harmelen ${ }^{41}$, Fred Hasselman ${ }^{42}$, Kevin D. Hochard ${ }^{43}$, Mark R. Hoffarth ${ }^{44}$, Nicholas P. Holmes ${ }^{45}$, Michael Ingre ${ }^{97}$, Peder M. Isager ${ }^{47}$, Hanna K. Isotalus ${ }^{48}$, Christer Johansson ${ }^{49}$, Konrad Juszczyk ${ }^{50}$, David A. Kenny ${ }^{51}$, Ahmed A. Khalil ${ }^{52,53,54}$, Barbara Konat ${ }^{55}$, Junpeng Lao ${ }^{56}$,

Erik Gahner Larsen ${ }^{57}$, Gerine M. A. Lodder ${ }^{58}$, Jiří Lukavský59, Christopher R. Madan ${ }^{45}$, David Manheim ${ }^{60}$, Stephen R. Martin ${ }^{61}$, Andrea E. Martin 21,62, Deborah G. Mayo63, Randy J. McCarthy ${ }^{64}$, Kevin McConway ${ }^{65}$, Colin McFarland66, Amanda Q. X. Nio67, Gustav Nilsonne ${ }^{68,69,70}$, Cilene Lino de Oliveira ${ }^{71}$, Jean-Jacques Orban de Xivry ${ }^{72}$,

Sam Parsons ${ }^{6}$, Gerit Pfuhl' ${ }^{73}$,

Kimberly A. Quinn ${ }^{74}$, John J. Sakon ${ }^{75}$,

S. Adil Saribay ${ }^{76}$, Iris K. Schneider ${ }^{77}$,

Manojkumar Selvaraju ${ }^{78,79}$, Zsuzsika Sjoerds ${ }^{80,81}$, Samuel G. Smith ${ }^{82}$, Tim Smits ${ }^{83}$,

Jeffrey R. Spies ${ }^{84,85}$, Vishnu Sreekumar ${ }^{86}$, Crystal N. Steltenpohl ${ }^{87}$, Neil Stenhouse ${ }^{88}$,

Wojciech Świątkowski ${ }^{89}$, Miguel A. Vadillo ${ }^{90}$, Marcel A. L. M. Van Assen ${ }^{91,92 \text {, }}$

Matt N. Williams ${ }^{93}$, Samantha E. Williams ${ }^{94}$, Donald R. Williams ${ }^{95}$, Tal Yarkoni ${ }^{30}$, Ignazio Ziano ${ }^{96}$ and Rolf A. Zwaan ${ }^{46}$ ${ }^{1}$ Human-Technology Interaction, Eindhoven University of Technology, Eindhoven,

The Netherlands. ${ }^{2}$ National Scientific and Technical Research Council (CONICET), Buenos Aires, Argentina. ${ }^{3}$ Max Planck Institute for Empirical Aesthetics, Frankfurt, Germany. ${ }^{4}$ Heymans Institute for Psychological Research, University of Groningen, Groningen, The Netherlands. ${ }^{5}$ College of Education, Psychology and Social Work, Flinders University, Adelaide, South Australia, Australia. ${ }^{6}$ Department of Experimental Psychology, University of Oxford, Oxford, UK. 'Department of Computer Science, Illinois Institute of Technology, Chicago, IL, USA. ${ }^{8}$ Department of Psychology, Nottingham Trent
University, Nottingham, UK. ${ }^{9}$ Faculty of Linguistics and Literature, Bielefeld University, Bielefeld, Germany. ${ }^{10}$ Psychology, University of Nevada, Las Vegas, Las Vegas, NV, USA. " University of Wisconsin-Madison, Madison, WI, USA. ${ }^{12}$ Psychology, Missouri State University, Springfield, MO, USA. ${ }^{13}$ Health, Human Performance, and Recreation, University of Arkansas, Fayetteville, AR, USA. ${ }^{14}$ Department of Development and Regeneration, KU Leuven, Leuven, Belgium.

${ }^{15}$ Department of Medical Statistics and Bioinformatics, Leiden University Medical Center, Leiden, The Netherlands. ${ }^{16}$ Department of Psychology, Linnaeus University, Kalmar, Sweden. ${ }^{17}$ Department of Human Development and Psychology, Tzu-Chi University, Hualien City, Taiwan. ${ }^{18}$ Department of Surgery, University of British Columbia, Victoria, British Columbia, Canada. ${ }^{19}$ Department of Psychology, University of Cambridge, Cambridge, UK. ${ }^{20}$ Centre for Statistics in Medicine, University of Oxford, Oxford, UK. ${ }^{21}$ Department of Psychology, School of Philosophy, Psychology and Language Sciences, The University of Edinburgh, Edinburgh, UK. ${ }^{22}$ School of Psychology, Bangor University, Bangor, UK. ${ }^{23}$ Institute of Neuroscience and Psychology, University of Glasgow, Glasgow, UK. ${ }^{24}$ Ramsey Decision Theoretics, Washington, DC, USA. ${ }^{25}$ Department of Behavioural Sciences and Learning, Linköping University, Linköping, Sweden. ${ }^{26}$ College of Social Work, Florida State University, Tallahassee, FL, USA. ${ }^{27}$ Departments of Psychology and Philosophy, Yale University, New Haven, CT, USA. ${ }^{28}$ Department of English, University of Louisiana at Lafayette, Lafayette, LA, USA. ${ }^{29}$ Department of Psychology, St. Edward's University, Austin, TX, USA. ${ }^{30}$ Department of Psychology, University of Texas at Austin, Austin, TX, USA. ${ }^{31}$ Department of Management, West Virginia University, Morgantown, WV, USA. ${ }^{32}$ Department of Psychology, Rutgers University, New Brunswick, NJ, USA. ${ }^{33}$ Department of Political Science, Indiana University Purdue University, Indianapolis, IN, USA. ${ }^{34}$ Booking.com, Amsterdam, The Netherlands. ${ }^{35}$ Department of English, American and Romance Studies, RWTH Aachen University, Aachen, Germany. ${ }^{36}$ School of Psychology, Keele University, Keele, UK. ${ }^{37}$ Centre of Excellence for Statistical Innovation, UCB Celltech, Slough, UK. ${ }^{38}$ Translational Neurosurgery, Eberhard Karls University Tübingen, Tübingen, Germany. ${ }^{39}$ International Centre for Ethics in Sciences and Humanities, Eberhard Karls University Tübingen, Tübingen, Germany. ${ }^{40}$ Department of Radiology, University of Cambridge, Cambridge, UK. ${ }^{41}$ Department of Psychiatry, University of Cambridge, Cambridge, UK. ${ }^{42}$ Behavioural Science Institute, Radboud University Nijmegen, Nijmegen, The Netherlands. ${ }^{43}$ Department of Psychology, University of Chester, Chester, UK. ${ }^{44}$ Department of Psychology, New York University, New York, NY, USA. ${ }^{45}$ School of Psychology, University of Nottingham, Nottingham, UK. ${ }^{46}$ Department of Psychology, Education, and Child Studies, Erasmus University Rotterdam, Rotterdam, The Netherlands.

${ }^{47}$ Department of Clinical and Experimental Medicine, 
University of Linköping, Linköping, Sweden. ${ }^{48}$ School of Clinical Sciences, University of Bristol, Bristol, UK. ${ }^{49}$ Occupational Orthopaedics and Research, Sahlgrenska University Hospital, Gothenburg, Sweden. ${ }^{50}$ The Faculty of Modern Languages and Literatures, Institute of Linguistics, Psycholinguistics Department, Adam Mickiewicz University, Poznań, Poland. ${ }^{51}$ Department of Psychological Sciences, University of Connecticut, Storrs, CT, USA. ${ }^{52}$ Center for Stroke Research Berlin, Charité Universitätsmedizin Berlin, Berlin, Germany. ${ }^{53}$ Max Planck Institute for Human Cognitive and Brain Sciences, Leipzig, Germany. ${ }^{54}$ Berlin School of Mind and Brain, Humboldt-Universität zu Berlin, Berlin, Germany. ${ }^{55}$ Social Sciences, Adam Mickiewicz University, Poznań, Poland. ${ }^{56}$ Department of Psychology, University of Fribourg, Fribourg, Switzerland. ${ }^{57}$ School of Politics and International Relations, University of Kent, Canterbury, UK. ${ }^{58}$ Department of Sociology/ICS, University of Groningen, Groningen, The Netherlands. ${ }^{59}$ Institute of Psychology, Czech Academy of Sciences, Prague, Czech Republic. ${ }^{60}$ Pardee RAND Graduate School, RAND Corporation, Arlington, VA, USA. ${ }^{61}$ Psychology and Neuroscience, Baylor University, Waco, TX, USA.

${ }^{62}$ Max Planck Institute for Psycholinguistics, Nijmegen, The Netherlands. ${ }^{63}$ Department of Philosophy, Virginia Tech, Blacksburg, VA, USA. ${ }^{64}$ Center for the Study of Family Violence and Sexual Assault, Northern Illinois University, DeKalb, IL, USA. ${ }^{65}$ School of Mathematics and Statistics, The Open University, Milton Keynes, UK. ${ }^{6}{ }^{6}$ Skyscanner, Edinburgh, UK. ${ }^{67}$ School of Biomedical Engineering and Imaging Sciences, King's College London, London, UK. ${ }^{68}$ Stress Research Institute, Stockholm University, Stockholm, Sweden. ${ }^{69}$ Department of Clinical Neuroscience, Stockholm, Sweden. ${ }^{70}$ Department of Psychology, Stanford University, Stanford, CA, USA. ${ }^{71}$ Laboratory of Behavioral Neurobiology, Department of Physiological Sciences, Federal University of Santa Catarina, Florianópolis, Brazil. ${ }^{72}$ Department of Kinesiology, KU Leuven, Leuven, Belgium. ${ }^{73}$ Department of Psychology, UiT The Arctic University of Norway, Tromsø, Norway. ${ }^{74}$ Department of Psychology, DePaul University, Chicago, IL, USA. ${ }^{75}$ Center for Neural Science, New York University, New York, NY, USA. ${ }^{76}$ Department of Psychology, Boğaziçi University, Istanbul, Turkey. ${ }^{77}$ Psychology, University of Cologne, Cologne, Germany. ${ }^{78}$ Saudi Human Genome Program, King Abdulaziz City for Science and Technology (KACST), Riyadh, Saudi Arabia. ${ }^{79}$ Integrated Gulf Biosystems, Riyadh, Saudi Arabia. ${ }^{80}$ Cognitive Psychology Unit, Institute of Psychology, Leiden University, Leiden, The Netherlands. ${ }^{81}$ Leiden Institute for Brain and Cognition, Leiden University, Leiden, The Netherlands. ${ }^{82}$ Leeds Institute of Health Sciences, University of Leeds, Leeds, UK. ${ }^{83}$ Institute for Media Studies, KU Leuven, Leuven, Belgium. ${ }^{84}$ Center for Open Science, Charlottesville, VA, USA.

${ }^{85}$ Department of Engineering and Society, University of Virginia, Charlottesville, VA, USA. ${ }^{86}$ Surgical Neurology Branch, National Institute of Neurological Disorders and Stroke, National Institutes of Health, Bethesda, MD, USA. ${ }^{87}$ Department of Psychology, University of Southern Indiana, Evansville, IN, USA. ${ }^{88}$ Life Sciences Communication, University of Wisconsin-Madison, Madison, WI, USA. ${ }^{89}$ Department of Social Psychology, Institute of Psychology, University of Lausanne, Lausanne, Switzerland. ${ }^{90}$ Departamento de Psicología Básica, Universidad Autónoma de Madrid, Madrid, Spain. ${ }^{91}$ Department of Methodology and Statistics, Tilburg University, Tilburg, The Netherlands. ${ }^{92}$ Department of Sociology, Utrecht University, Utrecht, The Netherlands. ${ }^{93}$ School of Psychology, Massey University, Auckland, New Zealand. ${ }^{94}$ Psychology, Saint Louis University, St. Louis, MO, USA. ${ }^{95}$ Psychology, University of California, Davis, Davis, CA, USA. ${ }^{96}$ Marketing Department, Ghent University, Ghent, Belgium. ${ }^{97}$ Unaffiliated: michael.ingre@gmail.com *e-mail:D.Lakens@tue.nl

Published online: 26 February 2018 https://doi.org/10.1038/s41562-018-0311-x

\section{References}

1. Benjamin, D. J. et al. Nat. Hum. Behav. 2, 6-10 (2018).

2. Wacholder, S., Chanock, S., Garcia-Closas, M., El Ghormli, L. \& Rothman, N. J. Natl Cancer Inst. 96, 434-442 (2004).

3. Open Science Collaboration Science 349, aac4716 (2015).

4. Senn, S. Statistical Issues in Drug Development 2nd edn (John Wiley \& Sons, Chichester, 2007).

5. Mayo, D. Statistical Inference as Severe Testing: How to Get Beyond the Statistics Wars (Cambridge Univ. Press, Cambridge, 2018)

6. Johnson, V. E., Payne, R. D., Wang, T., Asher, A. \& Mandal, S. J. Am. Stat. Assoc. 112, 1-10 (2017).

7. Chambers, C. D., Dienes, Z., McIntosh, R. D., Rotshtein, P. \& Willmes, K. Cortex 66, A1-A2 (2015).

8. Lyons, L. Preprint at http://arxiv.org/abs/1310.1284 (2013).

9. Field, S. A., Tyre, A. J., Jonzen, N., Rhodes, J. R. \& Possingham, H. P. Ecol. Lett. 7, 669-675 (2004).

10. Grieve, A. P. Pharm. Stat. 14, 139-150 (2015).

11. Mudge, J. F., Baker, L. F., Edge, C. B. \& Houlahan, J. E. PLoS ONE 7, e32734 (2012).

12. Skipper, J. K., Guenther, A. L. \& Nass, G. Am. Sociol. 2, 16-18 (1967).

13. Neyman, J. \& Pearson, E. S. Phil. Trans. R. Soc. Lond. Ser. A 231, 694-706 (1933)

14. Fisher R. A. Statistical Methods and Scientific Inferences (Hafner, Oxford, 1956).

15. Casadevall, A. \& Fang, F. C. mBio 7, e01902-16 (2016).
Acknowledgements

We thank D. Barr, F. Cheung, D. Colquhoun, H. IJzerman, H. Motulsky and R. Morey for helpful discussions while drafting this Comment. D.L. was supported by Nederlandse Organisatie voor Wetenschappelijk Onderzoek 452-17-013. F.G.A. was supported by CONICET. M.A.J.A. was funded by a Biotechnology and Biological Sciences Research Council AFL Fellowship (BB/M013596/1). G.S.C. was supported by the National Institute for Health Research Biomedical Research Centre, Oxford. Z.C. was supported by the Economic and Social Research Council (grant number C106891X). E.S.C. was supported by the European Research Council (ERC-2015-StG-677270). L.D. is supported by the European Research Council (ERC-2014-CoG-647910 KINSHIP). A.-L.v.H. is funded by a Royal Society Dorothy Hodgkin Fellowship (DH150176). M.R.H. was supported by the National Science Foundation under grant SBE SPRF-FR 1714446. J.Lao was supported by the Swiss National Science Foundation grant 100014_156490/1. C.L.d.O. was supported by Conselho Nacional de Desenvolvimento Científico e Tecnológico. A.E.M. was supported by the Economic and Social Research Council of the United Kingdom (grant number ES/K009095/1). J.-J.O.d.X. is supported by an internal grant from the KU Leuven (STG/14/054) and by the Fonds voor Wetenschappelijk Onderzoek (1519916N). S.P. was supported by the European Research Council (FP7/2007-2013; ERC grant agreement no. 324176). G.M.A.L. was funded by Nederlandse Organisatie voor Wetenschappelijk Onderzoek 453-14-016. S.G.S. is supported by a Cancer Research UK Fellowship (C42785/A17965). V.S. was supported by the National Institute for Neurological Disorders and Stroke Intramural Research Program (IRP) M.A.V. was supported by grant 2016-T1/SOC-1395 from Comunidad de Madrid. T.Y. was supported by National Institutes of Health award R01MH109682.

\section{Author contributions}

D.L., N.W.F., M.G.-M., J.A.G., N.P.H., A.A.K., S.R.M., V.S., S.D.B. and C.N.S. participated in brainstorming, drafting the Comment and data analysis. C.J.A., S.E.A., T.B., E.M.B., B.V.C., Z.C., G.S.C., S.D., D.J.D., B.D.E., J.D.F., J.G.F., A.-L.v.H., M.I., P.M.I., H.K.I., J.Lao, G.M.A.L., D.M., A.E.M., K.M., A.Q.X.N., G.N., C.L.d.O., J.-J.O.d.X., G.P, K.A.Q., I.K.S., Z.S., S.G.S., J.R.S., M.A.L.M.V.A., M.N.W., D.R.W., T.Y. and R.A.Z. participated in brainstorming and drafting the Comment. F.G.A., R.B.B., M.I.F., F.H. and S.P. participated in drafting the Comment and data analysis. M.A.J.A., D.E.B., S.-C.C., B.C., L.J.C., H.D., L.D., M.R.H., E.G.L., R.J.M., J.J.S., S.A.S., T.S., N.S., W.Ś. and M.A.V. participated in brainstorming. F.A., A.R.C., R.C., E.S.C., A.F, C.G., A.P.G., R.G., J.G., K.D.H, C.J., K.J, D.A.K., B.K., J.Lukavský, C.R.M., D.G.M., C.M., M.S., S.E.W. I.Z. did not participate in drafting the Comment because the points that they would have raised had already been incorporated, or they endorse a sufficiently large part of the contents as if participation had occurred. Except for the first author, authorship order is alphabetical.

Competing interests

The authors declare no competing interests. 